\title{
Opportunities, Challenges and Directions in Science and Technology for Tackling COVID-19
}

\author{
Appa Rao Podile ${ }^{1}$ D $\cdot$ Anirban Basu ${ }^{1}$
}

Received: 6 May 2020 / Accepted: 19 May 2020 / Published online: 26 May 2020

C Indian National Academy of Engineering 2020

\begin{abstract}
The ongoing global crisis due to Coronavirus disease-2019 (COVID-19) pandemic has caused an enormous socioeconomic burden. A novel coronavirus causing severe acute respiratory syndrome (SARS-CoV-2) that evolved from a virus infecting bats is responsible for COVID-19, first reported in the Chinese city of Wuhan. In the absence of any specific scientifically proven and clinically tested drug or vaccine against SARS-CoV-2, the virus is wreaking havoc across the world, claiming more than 2,50,000 lives in less than 5 months, and posed a global health emergency. The scientific community is relentlessly working on the design and testing of vaccines and antiviral drugs against the novel coronavirus, several of which have reached advanced stages of testing and are undergoing clinical trials. Here we discuss the recent advances and developments in understanding the etiology and epidemiology of the COVID-19 pandemic, the factors influencing the disease transmission, and the countermeasures adopted to combat and stop further spread of the disease.
\end{abstract}

Keywords COVID-19 $\cdot$ Coronavirus $\cdot$ Pandemic $\cdot$ SARS-CoV-2 $\cdot$ Disease triangle

\section{The COVID-19 Pandemic}

In an attempt to perpetuate, different organisms evolved different lifestyles, and one such lifestyle is to live on other organisms as a parasite or pathogen. The organisms attacked by the pathogens or parasites are referred to as hosts. The host and pathogen continue to learn from each other, wherein the pathogen tries to dominate the host and the host tries to suppress the pathogen, which can be compared to the newer strategies adopted by two competing nations to dominate each other.

In the evolutionary race between the host and pathogen, there is a continuous pressure on both the pathogen and the host to evolve continuously. In the process, humankind has witnessed several epidemics and pandemics. A new Coronavirus $(\mathrm{CoV})$, which causes severe acute respiratory syndrome (SARS-CoV), has been named as SARS-CoV-2 by the International Committee on Taxonomy of Viruses. The resulting disease is referred to as Coronavirus disease-2019

Appa Rao Podile

podilerao@gmail.com

1 Department of Plant Sciences, School of Life Sciences, University of Hyderabad, P.O. Central University Campus, Hyderabad, Telangana 500 046, India
(COVID-19) by World Health Organization (WHO), which has killed over 2,50,000 people in less than 5 months.

The COVID-19 is neither the first pandemic nor is going to be the last one. During the second, third, sixth and fourteenth centuries, when not much science has developed, about 250 million lives were lost due to plague. In the sixteenth century, the smallpox virus killed over 50 million people across the world. More recently, plague in the eighteenth century and the Spanish flu in the early twentieth century also took a heavy toll close to 200 million lives. The twentyfirst century witnessed its first pandemic caused by an influenza virus (H1N1 swine flu) that claimed 1,00,000-4,00,000 lives in the first year itself. The deaths due to COVID-19 could have been higher if there were no public health measures in place. Such pandemics will certainly continue in the years to come. Nevertheless, to contain such infectious agents and spread of disease, public health measures on the one hand and the availability of drugs/vaccines on the other, will minimize the casualties. The SARS-CoV-2, apparently evolved from a virus infecting the bats, is threatening to take lives of a large number of humans irrespective of the region, religion, ethnicity, wealth, gender, age (although people over the age of 65 and comorbid patients are likely to be more vulnerable), and such other considerations. There has been 
a larger question for the experts working with pathogens on how, when and why the pathogens evolve.

\section{Disease Triangle: Determines Epidemiological Outcomes}

The occurrence of a disease is dependent on three critical factors viz., pathogen, host and the environment, which pathologists define as the disease triangle, each one representing one of the three faces of the triangle. Intricate interactions and interplay between these three factors determine disease outcomes and implications on public health. The ability of a pathogen to cause the disease being primary, the degree of successful expression of the ability to cause disease plays a crucial role from the pathogen's side. Similarly, the susceptibility/resistance of the host plays a dominant role, while the predisposing factors (of the host) contribute to susceptibility. The often neglected third face of the triangle is the environment, in terms of how conducive it is to the host and the pathogen. If the pathogen has the ability to cause the disease, it may affect a few individuals. However, if its virulence is high, it has the power to kill several individuals-should the environment enable its transmission. For the SARS-CoV-2 to play havoc, the virulence of the strain (the one causing disease in an area) is to be high, and the environment has to be conducive, besides the availability of a susceptible host (in terms of over-aged population or comorbid in the known cases). If the host is resistant or not susceptible to the SARS-CoV-2 strain under circulation, the epidemic will inevitably fade away. If the environment is not favourable for the development of the disease, the pathogen will meet the same fate as in the case of a resistant host. The relation between these three components of the disease triangle looks so simple, but determines the extent of damage due to a disease like COVID-19.

The mysterious new illness is widely reported to have variation and severity as the distinctive and perplexing features. Scientists hypothesized differently to explain the variation. While many may look at the variation in the virus as a reason, there is a strong reason to look at the host genetics as a significant factor for this variation and severity. Most of them are asymptomatic, while others suffer severe damage to the lungs, which could be fatal. Many more are in the middle. The dramatic difference in the response of the people to this virus has been a major question that bothers everyone around. In the disease triangle, the interaction between partners (host and pathogen) is always considered as the most crucial step in deciding the outcome and severity of the infection. The host genetic differences can either increase or decrease a person's risk of certain diseases. Knowledge of the response of the host genes could help the development of both preventive (vaccines) and curative (drugs) measures for several diseases, including COVID-19.

\section{Understanding the Contagion: Structural and Genomic Profiling of the SARS-CoV-2}

The more we understand the contagion, the easier it is to develop measures to prevent or control the spread of disease. Identification and sequencing of the agent responsible for the COVID-19 outbreak showed that it was a novel CoV. The new virus shared $88 \%$ and $79.5 \%$ sequence identity with two bat-derived SARS-like CoV and SARS-CoV, respectively (Lu et al. 2020; Zhou et al. 2020a). The coronaviral genome encodes four major structural proteins: the spike (S) protein, nucleocapsid $(\mathrm{N})$ protein, membrane $(\mathrm{M})$ protein, and the envelope (E) protein (Schoeman and Fielding 2019). The $\mathrm{S}$ protein facilitates the entry of $\mathrm{CoV}$ into the target cell. Detailed analysis of the sequence of SARS-CoV-2 S protein showed that it was only $75 \%$ identical with the SARS-CoV $\mathrm{S}$ protein ( $\mathrm{Lu}$ et al. 2020; Zhou et al. 2020a). On the other hand, the receptor-binding motif (RBM) analyses indicated that the amino acids playing a role in receptor binding in the RBM are conserved. Based on these observations, Wan et al. (2020) suggested that the two CoV strains use the same host receptor to enter the target cells.

The structural and genetic make-ups of the SARS-CoV-2 have been analyzed using different approaches in just 4 months. It has about 30,000 ribonucleotides that constitute the single-stranded positive-sense RNA genome of this $\mathrm{CoV}$. A helical nucleocapsid protects the viral genome. It is covered by a spherical envelope embedded with the $\mathrm{E}, \mathrm{S}$, and M proteins (Schoeman and Fielding 2019) that appears like a crown under the electron microscope. Global initiatives such as the Global Initiative on Sharing Avian Influenza Data (GISAID) enable curation and real-time sharing of genome sequences of SARS-CoV-2 across the globe to provide a better understanding of the disease and evolutionary network of the virus. Forster et al. (2020) analyzed the genome of 160 SARS-CoV-2 sequences at GISAID as of 4th March 2020. The SARS-CoV-2 sequence has a 96\% similarity with the bat coronavirus, as was known from an earlier study (Wu et al. 2020). There are three central variants (A being the ancestral type, $\mathrm{B}$, and $\mathrm{C}$ ), distinguished by amino acid changes. Interestingly, the B type is most common in East Asia. In contrast, the A and C types are predominantly found outside East Asia (Europe and America), which points to an immunological or environmental resistance against B type outside East Asia.

Another global initiative in the name of COVID-19 Host Genetics Initiative (CHGI), which is at infancy, is deeply engaged in explaining the role of the host genome in severity and susceptibility to COVID-19. The success of such 
initiatives heavily depends on the cooperation of individuals to consent and willingly share the requisite biomedical information for research purposes.

The newly evolved SARS-CoV-2, traced to Wuhan in China, is behaving differently in different populations. The most recent analysis of the 3636 sequences (from 55 countries) of SARS-CoV-2, available at GISAID as on 6th April 2020, was carried out by the National Institute of Biomedical Genomics (NIBG), India. The results indicated that at least ten types of the virus evolved from the ancestral Wuhan strain (O type) in the last 4 months. The A2a type that evolved towards the end of March 2020, probably replaced others and evolved as the dominant type. SARS-CoV-2 being a virus with single-stranded RNA as its genome, is less stable, particularly in the absence of a proof-reading mechanism during replication in the host tissue. It is not entirely clear whether the mutations in the genome of SARS$\mathrm{CoV}-2$ or 'some other' factors are responsible for the virulence and rapid spread of COVID-19, as an adequate number of genomes from all parts of the world has not been analyzed sufficiently. Large scale analysis of the sequence information, with increased global efforts, will provide unique vaccine markers with specific target(s) and also helps in designing vaccines against different types of the COVID-19.

Sequence information of Indian strains of SARS-CoV-2 (scanty in the public domain), available now due to efforts by Indian scientists, requires a thorough analysis to understand the origin and similarity/uniqueness in comparison with the $\mathrm{A}, \mathrm{B}$ and $\mathrm{C}$ variants. A mere change in a nucleotide (which can happen for different reasons) cannot be treated as mutation. If a stable change occurs in the nucleotide sequence and is passed on to the next generation, it is referred to as a mutation. Further, a mutation need not always drastically affect the virulence of the virus, unless the mutation has occurred in a most crucial portion of the genome. India has to analyze a few hundreds of sequences from the COVID19 strains in different geographical locations and link to the suspected origin of infection from the patients across India to understand if any mutations were affecting the virulence. Once we trace the sources of undocumented COVID-19 infection, the evolutionary networks can help to prevent a recurrent spread of the disease.

\section{Development of Countermeasures Against COVID-19}

For centuries, scientists have found solutions to several dreaded diseases caused by invisible microorganisms like bacteria, fungi, and viruses, which otherwise took heavy tolls. They were successful with the two major approaches, prevention or control, to save lives. Antibiotics or synthetic drugs were discovered to control, and vaccines were developed to prevent. Science has not yet reached a stage to predict the evolution of the pathogens accurately. Therefore, only when pandemics break out, they start working for solutions.

Developments in life sciences with the discovery of the double-helical structure of DNA and subsequent technologies associated with the nucleic acid on the one hand and the availability of high-performance computing facility that can analyze several gigabytes of sequence information in a short time on the other, paved way to significantly reduce the time required for drug discovery or vaccine development. A century ago, in a similar pandemic, it took almost 20 years for the vaccine to be made available. The disease was managed by herd immunity in the absence of drugs and vaccines. Recently, the Ebola vaccine was developed in 5 years, and Zika vaccine was developed in less than 2 years. However, for COVID-19 the vaccine may be available in a record time of about 12 months more due to international collaboration, rather than competition. In the meanwhile, there would be a few promising drugs repurposed.

Over 100 vaccine candidates against COVID-19 agent are at various stages of development. At least seven of them are in Phase 1 clinical trials. China, Germany and the USA are testing two vaccines each, and one vaccine from the UK is also under clinical trials. China is moving to Phase 2 clinical trials, at least for one vaccine. The vaccine being developed by Moderna Inc and backed by the US National Institutes of Health (NIH) could be the front runner. The vaccine being developed by Jenner Institute of the University of Oxford is aggressively pushing to make at least one million doses of vaccine available by September 2020. In India, the immunomodulatory Mycobacterium w (Mw) vaccine, developed initially to treat leprosy, is being repurposed as an adjunct to treat critically ill COVID-19 patients in clinical trials. It is possible that most of the international efforts may succeed in fight COVID-19 with an efficient vaccine by early 2021 . These vaccines, however, necessarily need to prove safety and efficacy against COVID- 19.

Experimental evidence indicates that the Bacille Calmette-Guérin (BCG) vaccine (primarily used against tuberculosis disease) may have some non-specific effects on the immune system. However, neither these effects have been well characterized, nor were validated in clinical trials. There is not enough evidence that the BCG vaccine can provide immunity against the COVID-19 virus infection. Clinical trials addressing this question are underway. WHO has not recommended BCG vaccination to prevent COVID19 as it awaits evidence through scientific evaluation.

To devise viable therapeutic strategies, it is essential to understand the biology of the virus-its detailed structure, the way it causes disease (pathogenesis), the way it infects people, and the way it progresses and, more importantly, the way it interacts with the host. The past experience suggests 
that understanding the key cellular factors used by an infectious agent for entry into the host can provide insights into disease transmission and also reveal therapeutic targets. The entry of coronaviruses, in general, into the cells depends on the binding of the viral $\mathrm{S}$ proteins to cellular receptors. The process of binding of the pathogen-derived molecules to the cellular receptors happens in a precise lock and key model. In the SARS-CoV-2, the process is initiated by the $S$ protein priming by host cell proteases. Hoffmann et al. (2020) elegantly showed that SARS-CoV-2 uses the SARS$\mathrm{CoV}$ receptor Angiotensin-Converting Enzyme-2 (ACE-2) for entering host cells (also reported by Zhou et al. 2020a) and the cellular serine protease TMPRSS-2 for priming of the $\mathrm{S}$ protein. As expected, the clinically proven TMPRSS-2 inhibitor camostat mesylate could block the viral entry and might be a potential target for therapeutic intervention.

Important proteins encoded by the virus could become the best drug targets. The main protease, one of the wellcharacterized drug targets in the case of coronaviruses, is essential for the processing of polyproteins translated from the viral RNA, along with the papain-like proteases (Anand et al. 2003; Hilgenfeld 2014). In a classic example, Zhang et al. (2020a) obtained the X-ray crystal structures of the unliganded SARS-CoV-2 main protease and its complex with an $\alpha$-ketoamide inhibitor which was developed into an effective inhibitor of the main protease. The pharmacokinetic characterization of the optimized inhibitor revealed the suitability of the compound by the inhalative route. The approach, by and large, has been the most rapid way to design a drug.

Scientists are relentlessly working on the design and testing of antivirals against SARS-CoV-2 in animals. Immunodiagnostic and immunotherapeutic strategies are being tried out against COVID-19, with a handful of them reaching advanced stages of testing. Selected antimalarials and antivirals, which could interfere with the function of one or the other important proteins of the SARS-CoV-2, are being repurposed to find the most appropriate drugs to treat COVID-19. SARS-CoV-2 can possibly be restricted by drugs previously used for other RNA viruses. Trials are currently underway with a combination of two antiretroviral drugs: lopinavir and ritonavir (used to treat HIV-AIDS). Other combinations with antimalarial drugs chloroquine and hydroxychloroquine (the latter reported to be more potent in treating COVID-19 by Yao et al. 2020) are also being tested for clinical usage. Apart from these, several potential therapeutic approaches have been reported for tackling COVID-19, including repurposed antiviral agents like remdesivir (drug against Ebola virus), favipiravir, EIDD-2801; corticosteroids; anticoagulants like heparin; human monoclonal antibodies such as bevacizumab, tocilizumab, and sarilumab; immunoenhancers like interferons, intravenous gamma globulin, natural killer (NK) cells, etc. (Zhang et al. 2020b; Zhou et al. 2020b), the efficacy and specificity of which needs to be validated through intensive research and clinical trials.

Transfusion of convalescent plasma from recovered patient donors has proved to be effective in treating severe and critically ill COVID-19 patients, without any severe adverse effects (Duan et al. 2020). However, convalescent plasma therapy requires further validation with large-scale randomized trials.

\section{Management of the Disease Depends Heavily on Scientific Data}

We have witnessed several catastrophes and global crises, and the way governments handled them with inputs from the science and technology sectors. In the absence of tested chemical/antibiotic treatments, interventions by the governments have focused on contact tracing, quarantine, social distancing and related measures. An assumption that intensive public measures can eradicate the SARS-CoV-2 seems unlikely, as per the public health authorities. It is possible that SARS-CoV-2 could circulate seasonally after the initial wave. Reliable mathematical models have projected the transmission dynamics of SARS-CoV through the pandemic and post-pandemic periods. At least three major factors seem to be regulating the SARS-CoV-2 transmission dynamics, viz., degree of seasonal variation of transmission, the duration of immunity, and the degree of cross-immunity between SARS-CoV-2 and other coronaviruses (Kissler et al. 2020). To mitigate the possibility of resurgences of COVID-19, the lessons learned during the first cycle of infection, especially on social distancing, need to be continued at least intermittently if not for prolonged periods. Failure to understand the transmission of COVID-19 and handle the first and the recurring cycles of disease, especially in a world that has been reduced to a 'global village' due to internationalization, will attract heavy penalties.

\section{Concluding Remarks}

Global emergencies like the ongoing COVID-19 pandemic can cause an enormous socioeconomic burden. Experience from developed and well-resourced nations with advanced healthcare systems such as the United States, China, Russia, United Kingdom and other European states like Spain, Italy, France and Germany demonstrates the nature of the havoc caused by the COVID-19 pandemic. These nations were severely hit by the pandemic and witnessed the maximum number of casualties. Pandemics also exert unprecedented pressure on developing countries like India that have inadequate financial capabilities or lack of healthcare resources. 
Research, though made rapid progress in the past 5 months, is still in infancy and will require considerable time and funds to translate into therapies and vaccines. Progress in science and technology can confidently address such challenges. Until clinically tested vaccines and specific antiviral agents for preventing and treating COVID-19 arrive in the market, we should keep on practising social distancing, avoid unnecessary travel and movement, and take utmost care in protecting ourselves by adhering to the guidelines and public health advisories issued from time to time by the WHO and various government agencies.

Acknowledgements The authors acknowledge Department of Science and Technology (DST), Government of India (GoI) for J.C. Bose Fellowship to ARP and University Grants Commission (UGC) for SRF fellowship to AB.

Author contributions ARP conceptualized the idea for the article, performed literature search and data analysis, and wrote the first draft of the manuscript. AB performed literature search, wrote portions of the manuscript and critically revised the work. Both authors read and approved the final manuscript.

Funding This work was supported by Department of Science and Technology (DST), Government of India (GoI), and University Grants Commission (UGC), respectively, in the form of J. C. Bose Fellowship to $\mathrm{ARP}$ and research fellowship to $\mathrm{AB}$. The funders had no role in study design, data collection and analysis, decision to publish, or preparation of the manuscript.

\section{Compliance with Ethical Standards}

Conflict of interest The authors declare that they have no conflicts of interest or competing interests.

\section{References}

Anand K, Ziebuhr J, Wadhwani P, Mesters JR, Hilgenfeld R (2003) Coronavirus main proteinase (3CLpro) structure: basis for design of anti-SARS drugs. Science 300(5626):1763-1767

Duan K, Liu B, Li C, Zhang H, Yu T, Qu J et al (2020) Effectiveness of convalescent plasma therapy in severe COVID-19 patients. Proc Natl Acad Sci USA 117(17):9490-9496

Forster P, Forster L, Renfrew C, Forster M (2020) Phylogenetic network analysis of SARS-CoV-2 genomes. Proc Natl Acad Sci USA 117(17):9241-9243
Hilgenfeld R (2014) From SARS to MERS: crystallographic studies on coronaviral proteases enable antiviral drug design. FEBS J 281(18):4085-4096

Hoffmann M, Kleine-Weber H, Schroeder S, Krüger N, Herrler T, Erichsen S et al (2020) SARS-CoV-2 cell entry depends on ACE2 and TMPRSS 2 and is blocked by a clinically proven protease inhibitor. Cell 181(2):271-280

Kissler SM, Tedijanto C, Goldstein E, Grad YH, Lipsitch M (2020) Projecting the transmission dynamics of SARS-CoV-2 through the postpandemic period. Science. https://doi.org/10.1126/scien ce.abb5793

Lu R, Zhao X, Li J, Niu P, Yang B, Wu H et al (2020) Genomic characterization and epidemiology of 2019 novel coronavirus: implications for virus origins and receptor binding. Lancet 395(10224):565-574

Schoeman D, Fielding BC (2019) Coronavirus envelope protein: current knowledge. Virol J 16(1):69

Wan Y, Shang J, Graham R, Baric RS, Li F (2020) Receptor recognition by the novel coronavirus from Wuhan: an analysis based on decade-long structural studies of SARS coronavirus. J Virol 94(7):e00127-e220. https://doi.org/10.1128/JVI.00127-20

Wu F, Zhao S, Yu B, Chen YM, Wang W, Song ZG et al (2020) A new coronavirus associated with human respiratory disease in China. Nature 579:265-269

Yao X, Ye F, Zhang M, Cui C, Huang B, Niu P et al (2020) In vitro antiviral activity and projection of optimized dosing design of hydroxychloroquine for the treatment of severe acute respiratory syndrome coronavirus 2 (SARS-CoV-2). Clin Infect Dis. https:// doi.org/10.1093/cid/ciaa237

Zhang L, Lin D, Sun X, Curth U, Drosten C, Sauerhering L et al (2020a) Crystal structure of SARS-CoV-2 main protease provides a basis for design of improved $\alpha$-ketoamide inhibitors. Science 368(6489):409-412

Zhang J, Xie B, Hashimoto K (2020b) Current status of potential therapeutic candidates for the COVID-19 crisis. Brain Behav Immun. https://doi.org/10.1016/j.bbi.2020.04.046

Zhou P, Yang XL, Wang XG, Hu B, Zhang L, Zhang W et al (2020a) A pneumonia outbreak associated with a new coronavirus of probable bat origin. Nature 579:270-273

Zhou M, Zhang X, Qu J (2020b) Coronavirus disease 2019 (COVID19): a clinical update. Front Med. https://doi.org/10.1007/s1168 4-020-0767-8

Publisher's Note Springer Nature remains neutral with regard to jurisdictional claims in published maps and institutional affiliations. 